\title{
Risk Evaluation of Environmental Carcinogens
}

\author{
Shigeaki SATO \\ Department of Hygiene, Kobe University School of Medicine
}

\begin{abstract}
Risk Evaluation of Environmental Carcinogens: Shigeaki Sato. Department of Hygiene. Kobe University School of Medicine - Quantitative risk evaluation of environmental carcinogens is required not only for their regulation but also for primary cancer prevention. Risk evaluation of carcinogens is carried out through identification of carcinogenicity, exposure assessment, dose-response assessment, and risk characterization. Exposure assessment is made by measuring the amounts of carcinogens in the environment and their human intake is estimated. Dose-response assessment is based upon whole-life carcinogenesis experiments in animals with multiple doses of carcinogens. By downward extrapolation of the dose-response curve obtained, a virtually safe dose (VSD), i.e., a dose which yields a cancer incidence of $10^{-6}$, is calculated and this value is compared with the actual human intake of the carcinogen concerned. When the human intake is around or less than the VSD, that environmental carcinogen is regarded safe to humans because humans as well as experimental animals develop cancer in almost $100 \%$ of cases during their whole life and the contribution of a chemical to the occurrence of cancer at an incidence of less than one out of one million is regarded as meaningless. These procedures for risk evaluation of carcinogenic food additives such as butylated hydraxyanisole and saccharin sodium showed that their human intake is one hundredth or much less than the respective VSD values, and this may be the case with most environmental carcinogens except some carcinogenic medical drugs. Human cancer development seems much more influenced by life-style factors, such as a high-fat diet or cigarette smoking, and recognition of this fact is crucial for primary cancer prevention.
\end{abstract}

(J Occup Health 1996; 38: 149-154)

Key words: Risk assessment, Exposure assessment, Dose-response assessment, Virtually safe dose, Regulation, Environmental carcinogens, Food

Received Nov 29, 1995; Accepted April I, 1996 Correspondence to: S. Sato, Department of Hygiene, Kobe University School of Medicine, 7-5-1, Kusunokicho, Chuo-ku, Kobe 650, Japan additives, Primary prevention, Modifying factors

In most countries, the actual numbers of cancer deaths are increasing and cancer has become the commonest cause of death. It increases with age. In people over the age of 70 years, the detection rate for cancer, including latent ones at autopsy, is reported to be around $40 \%{ }^{1)}$. This suggests that in old people the incidence rate for cancer, including very small latent ones unable to be detected even at autopsy could reach nearly $100 \%$. The incidence of spontaneous tumors has also been reported to be 70 to $100 \%$ in some strains of rats which were observed for more than two years ${ }^{2,3)}$. However, in humans the incidence of cancer of each organ varies chronologically in many countries. Some cancers are increasing but others are decreasing. The incidence also changes with immigration. These changing cancer patterns suggest that human cancer development is closely related to life style $\mathrm{e}^{4)}$. Actually, in our environment many factors have been found which are involved in human cancer development or which cause animal cancers when given in large amounts.

Recent development of short-term assay methods for probable carcinogens and extensive animal experiments have detected hundreds of environmental carcinogenic agents including those found in food, air, water, medical drugs and in the working place. A carcinogen is defined as a substance which either significantly increases the tumor incidence or significantly decreases the latent period of spontaneous tumor development in animals or humans ${ }^{5)}$. Thus, the actual effect of carcinogens, which have only been shown to cause cancer in experimental animals, on human cancer development is a big concern. This is especially so in cases where the substances concerned are found in workplaces or at other sites which can be legally regulated. Even if they are not subject to regulation, such as those carcinogens formed during food cooking or found naturally, their risk evaluation is crucial for the 
control of human cancer development.

\section{Legal regulation of hazardous chemicals in Japan}

Regulation of hazardous chemicals is carried out by different ministries, depending upon the difference in their category, as shown in Table $1^{6)}$. For example, food additives are regulated by the Food Sanitation Law of the Ministry of Health and Welfare, and chemicals in the workplace are regulated by the Industrial Safety and Health Law of the Ministry of Labor. For the elimination of hazardous chemicals, the most efficient way is to check their biological activities, including carcinogenicity, and to ban the agents before they become commercially available if they have been proven to be hazardous. Before the approval of new chemicals, their risk evaluation is therefore carried out by expert committees organized by the respective ministries according to respective laws through a review of physicochemical and biological data presented for the chemicals concerned. This is sometimes the case also with chemicals already available to the public, which have not been fully evaluated for their safety. For the evaluation, biological data such as toxicity, teratogenicity, mutagenicity or carcinogenicity are required. Among these data mutagenicity and carcinogenicity are regarded as crucial, especially for the risk evaluation of food additives after the issue of AF-2 [2-(2-furyl)-3-(5-nitro2-furyl) acrylamide] which was proven to be carcinogenic to animals after the establishment of mutagenicity in bacteria ${ }^{7}$. The guidelines for risk evaluation of chemicals, including these biological activities, have been made not only under the Food Sanitation Law but also under the Pharmaceutical Law or other laws ${ }^{8)}$. Details and examples of the regulation of food-related hazardous chemicals ${ }^{6}$ ) and workplace chemicals ${ }^{9)}$ have been published.

\section{Risk evaluation of carcinogens}

Among hazardous chemicals, carcinogens, i.e., substances which can induce cancer either in ani- mals or humans, draw much attention and should be carefully evaluated for their risk to humans. Among environmental chemicals or intermediates of products in commercial use, those which have genotoxic activity such as mutagenicity, those suspected of being human carcinogens epidemiologically or those used in large amounts for a long time by humans but not fully evaluated for their safety, are subject to carcinogenicity tests with experimental animals. The problem is the management of chemicals which have been proven to be carcinogenic. $\mathrm{AF}-2$, which was proven to be carcinogenic in mice in 1974, was immediately banned solely because of its carcinogenicity. However, most of the chemicals industrially synthesized and available to the public, such as food additives, pesticides, herbicides and medical drugs, are made to help humans to live safer or healthier lives. Regulation of these chemicals, after their carcinogenicity has been found, should therefore be done while carefully considering the balance between their risks and benefits. When non-carcinogenic alternatives are available, to ban carcinogenic agents is easy, but this is not always the case. Here, scientific and quantitative risk evaluation of carcinogens is crucial.

Risk evaluation of carcinogenic chemicals is made in the following four phases, as with other hazardous chemicals: indentification of carcinogenicity, exposure assessment, dose-response assessment and risk characterization ${ }^{10)}$. With those agents already proven to be carcinogenic to animals, assessment of human exposure levels and responses to very low doses to which humans are usually exposed, is crucial. Exposure assessment is made by measuring the amounts of respective carcinogens in the diet, air, water or other aspects of the environment, and their human intake is estimated. Because the presence of most carcinogens in our environment is usually very minimal, sensitive and reliable methods of detection of these chemicals are obligatory. As for food additives, the results of their assay in various foodstuffs and their daily intake by

Table 1. Laws which regulate chemical substances in Japan ${ }^{6)}$

\begin{tabular}{|c|c|c|}
\hline Chemical & Law & Ministry \\
\hline Food additives and contaminants & Food Sanitation Law & Health and Welfare \\
\hline Drugs and cosmetics & Pharmaceutical Law & Health and Welfare \\
\hline Poisons & Poisonous and Deleterious Substances Control Law & Health and Welfare \\
\hline Pesticides & Agricultural Chemicals Control Law & Agriculture, Forestry and Fishery \\
\hline Feed additives & Feed Safety Law & Agriculture, Forestry and Fishery \\
\hline Fertilizers & Fertilizers Control Law & Agriculture, Forestry and Fishery \\
\hline Industrial chemicals & $\begin{array}{l}\text { Law Concerning the Examination and Regulation of } \\
\text { Manufacture, etc., of Chemical Substances }\end{array}$ & $\begin{array}{l}\text { International Trade and Industry } \\
\text { Health and Welfare }\end{array}$ \\
\hline Workplace chemicals & Industrial Safety and Health Law & Labor \\
\hline
\end{tabular}


Japanese people, calculated by various methods, are published ${ }^{11)}$. Among these food additives, probable daily intakes of the carcinogenic agents, butylated hydroxyanisole (BHA) ${ }^{12}$, butylated hydroxytoluene $(\mathrm{BHT})^{13)}$ and saccharin sodium ${ }^{14)}$ are shown to be $0.216,0.273$ and $10.4 \mathrm{mg}$ per capita, respectively.

The next step in risk evaluation is the estimation of the cancer incidence at these very low levels of intake for an entire lifetime. This is done in animal carcinogenesis experiments by entire lifetime feeding (2 years for mice and rats) of carcinogens. Most animal carcinogenesis experiments have been done with a single dose or two doses of the carcinogen. In this case only a $\mathrm{TD}_{50}$ (tumor dose 50 ) value, which is a dose to induce tumors in $50 \%$ of the animals ${ }^{15}$, can be calculated but the response at very low doses is hard to estimate because the dose response curve of carcinogens is usually sigmoidal. Accordingly, to estimate the response at very low levels, multiple-dose experiments and calculation of the tumor incidence at these levels by downward extrapolation is necessary. The dose-response curve of non-carcinogenic hazardous chemicals is regarded as having a threshold which is defined as the no observed effect level (NOEL), as shown is Fig. 1. As for the legal regulation of these chemicals, an acceptable daily intake (ADI) is obtained by dividing the NOEL value by a safety factor (usually 100 or 500). In case of carcinogenic chemicals, however, if a threshold of dose response is considered to be present, very small amounts, such as those to which humans are usually exposed, and much less than the threshold, are regarded as safe, and this may be the case with almost all the environmental carcinogens. Therefore, in recent years and especially for regulatory purposes, the dose response

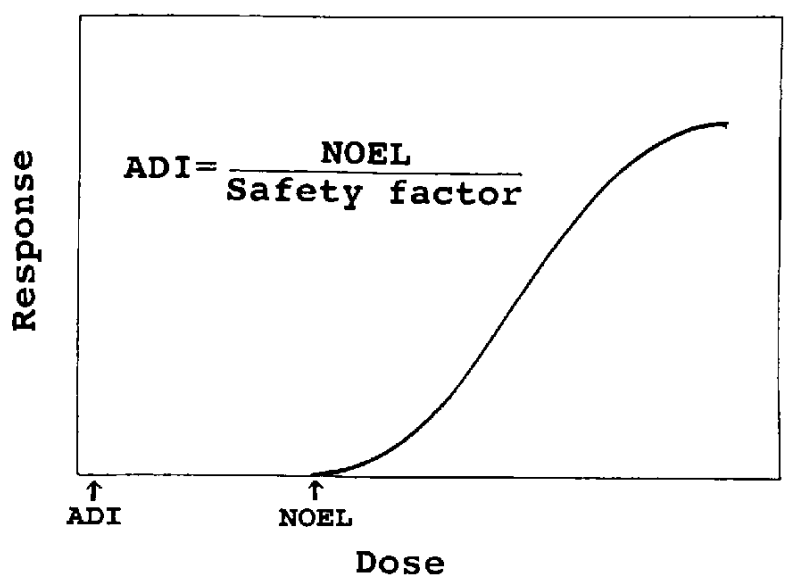

Fig. 1. Dose-response curve of non-carcinogenic hazardous chemicals. NOEL: no observed effect level, ADI : acceptable daily intake. curve of carcinogens is considered to be that shown in Fig. 2, converging to the origin without threshold, although a biological threshold may exist even for carcinogens. Such a curve is drawn theoretically from the data obtained actually with multipledose experiments. Based on this theoretically obtained curve, the dose which gives a tumor incidence of $10^{-6}$ is calculated by downward extrapolation (Fig. 2). This dose is called the virtually safe dose (VSD) ${ }^{16)}$, because that which yields tumors only in one of one million animals during their entire lifespan is regarded as virtually safe, compared with the nearly $100 \%$ cancer incidence during the whole life of either experimental animals or humans. The human intake of carcinogens is then compared with the VSD values thus obtained by conducting animal experiments. If the human intake of a particular carcinogen is less than or around the corresponding VSD value, the carcinogen exposure is regarded as safe to humans. This method of risk evaluation is based on the premise that the whole-life experimental period of two years is equivalent to the average life expectancy of humans of 70 years and that the daily intake of carcinogens per unit body weight in both animals and humans is comparable. Differences between animals and humans in susceptibility to carcinogens and in body mass are not considered. Animal experiments to obtain VSD values are very laborious and take a long time. Moreover, depending upon the difference in the method of calculating VSD, the values obtained often vary significantly ${ }^{17}$. Despite these drawbacks, the risk evaluation of carcinogens based on VSD values is now regarded as practical and is applied in various areas. In Fig. 3, VSD values for some carcinogens are shown ${ }^{17,18)}$. Aflatoxin $B_{1}$ is the strongest carcinogen and saccharin sodium and nitrilotriacetic acid are the weakest carcinogens. It should be noted that between these chemicals there

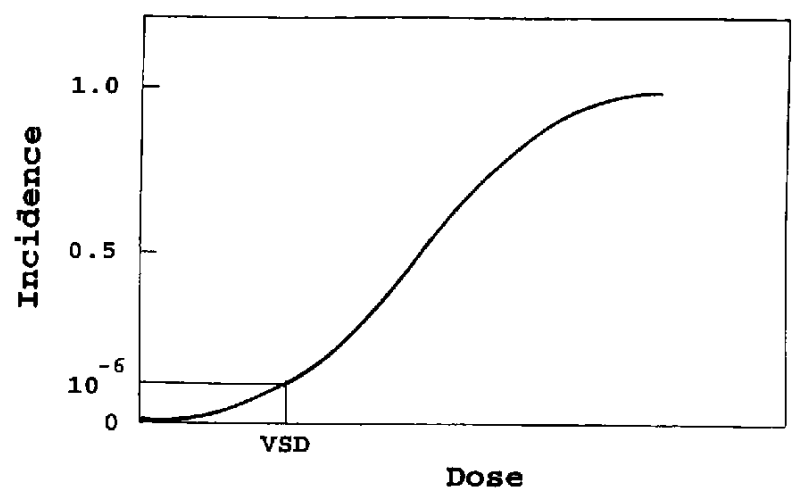

Fig. 2. Dose-response curve of carcinogens and virtually safe dose (VSD). 


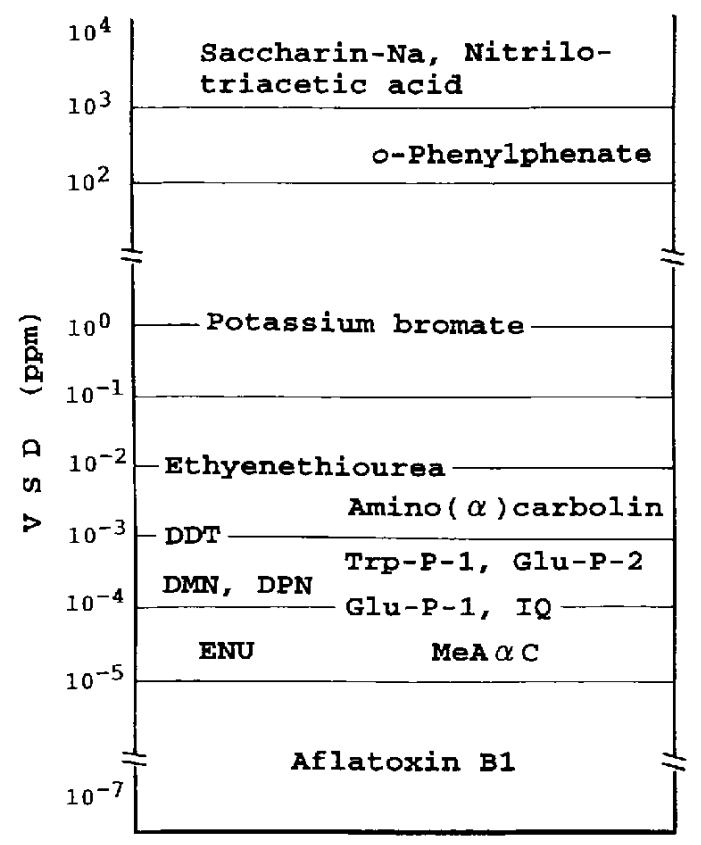

Fig. 3. VSD values of various carcinogens expressed as a concentration in diet or drinking water ${ }^{17,} 18$.

is a huge difference of a carcinogenic potency of $10^{10}$ although both groups are called environmental carcinogens. The VSD of potassium bromate, which is a carcinogenic food additive used for maturation of flour for bread making, has been calculated to be around $1 \mathrm{ppm}$ in drinking water ${ }^{17)}$. Carcinogenic heterocyclic amines present in cooked food, such as amino- $\alpha$-carboline, 3 -amino- 1,4 dimethyl-5H-pyrido $[4,3-b]$ indole (Trp-P-1), 2aminodipyrido $[1,2-a: 3$ ', 2'-d] imidazole (Glu-P2), 2-amino-6-methyldipyrido $\left[1,2-a: 3^{\prime}, 2^{\prime}-d\right]$ imidazole (Glu-P-1), 2-amino-3-methylimidazo $[4,5$ -f] quinoline (IQ) and methylamino- $\alpha$-carboline (MeA $\alpha \mathrm{C})^{19)}$ show VSD values of around $10^{-4}$ ppm in the diet, which are close to those for dimethylnitrosamine (DMN), dipropylnitrosamine (DPN) and ethylnitrosourea (ENU). Although not shown in this figure, the VSD value for BHA has been estimated as around $4.4 \mathrm{ppm}$ in the diet from the multiple-dose carcinogenesis experiments ${ }^{20}$.

\section{Risk characterization of some carcinogens}

Table 2 shows the average intakes of the carcinogenic food additives, BHA and saccharin sodium, by Japanese people and their respective VSD values, both expressed as $\mu \mathrm{g} / \mathrm{kg}$ b.w./day. The average human intake of BHA is one hundredth of its VSD and that of saccharin sodium is only one five hundredth of the VSD. These results clearly show that intake of carcinogenic food additives such as BHA
Table 2. Risk evaluation of carcinogenic food additives, BHA and saccharin sodium

\begin{tabular}{lcc}
\hline & BHA & Saccharin sodium \\
\hline Intake $(\mu \mathrm{g} / \mathrm{kg} \mathrm{b}$. w. $/$ day $)$ & $4.3^{\mathrm{a}}$ & $208^{\mathrm{a}}$ \\
VSD $(\mu \mathrm{g} / \mathrm{kg} \mathrm{b}$ b. w. day $)$ & $440^{\mathrm{b}}$ & $10^{5 \mathrm{c}}$ \\
\hline${ }^{\mathrm{a}}$ ref. $11,{ }^{\mathrm{b}}$ ref. $20,{ }^{\mathrm{c}}$ refs. $17,18$. &
\end{tabular}

and saccharin sodium does not at all contribute to the development of common human cancers. This is one of the reasons why the use of these carcinogenic food additives is permitted in Japan with restriction $^{6,21)}$. It is also the case of potassium bromate, the use of which up to $30 \mathrm{ppm}$ is permitted in wheat flour for bread, if this chemical is decomposed in the final products ${ }^{6)}$. With regard to most other carcinogenic food additives, or other categories of environmental carcinogens, their actual human intake may be less than their VSD values. This means that any common human cancers cannot be attributable to any specific environmental carcinogen. Of course some specific human cancers have been shown to be related to some specific carcinogens. These are summarized as group 1 (carcinogenic to humans) by the working group organized by the International Agency for Research on Cancer (IARC) (Table 3) ${ }^{22)}$. Among theses agents, carcinogens or conditions related to the workplace, such as aluminum production, 4-aminobiphenyl, manufacture of auramine, benzene, boot and shoe manufacture, coal gasification, coke production, iron and steel founding, mustard gas, the rubber industry, and so on are marked. Heavy exposure to these factors is, however, now seldom encountered at workplaces due to the improvement in conditions. Another category which should be noted is medical drugs including antitumor agents such as myleran, chlorambucil, cyclophosphamide, melphalan and MOPP, immunosuppressive agents such as azathioprine, and hormones such as diethylstilboestrol and oestrogens. Human growth hormone, used for the treatment of drawfism, has also been shown to induce leukemia in children ${ }^{23)}$. With the steady increase in the cure rate for various malignancies, the incidence of secondary tumors due to the antitumor agents is also increasing and care must be taken when these drugs are used for a long time and in a large amount. This is also the case with the use of immunosuppressive agents for organ transplantation. However, the proportion of human cancers attributable to the group 1 carcinogens shown above may be very small, probably less than one percent of all cancers. Even when all of the environmental carcinogens so far detected in animal experiments are taken together, the proportion of 
Table 3. Agents carcinogenic to humans ${ }^{22)}$

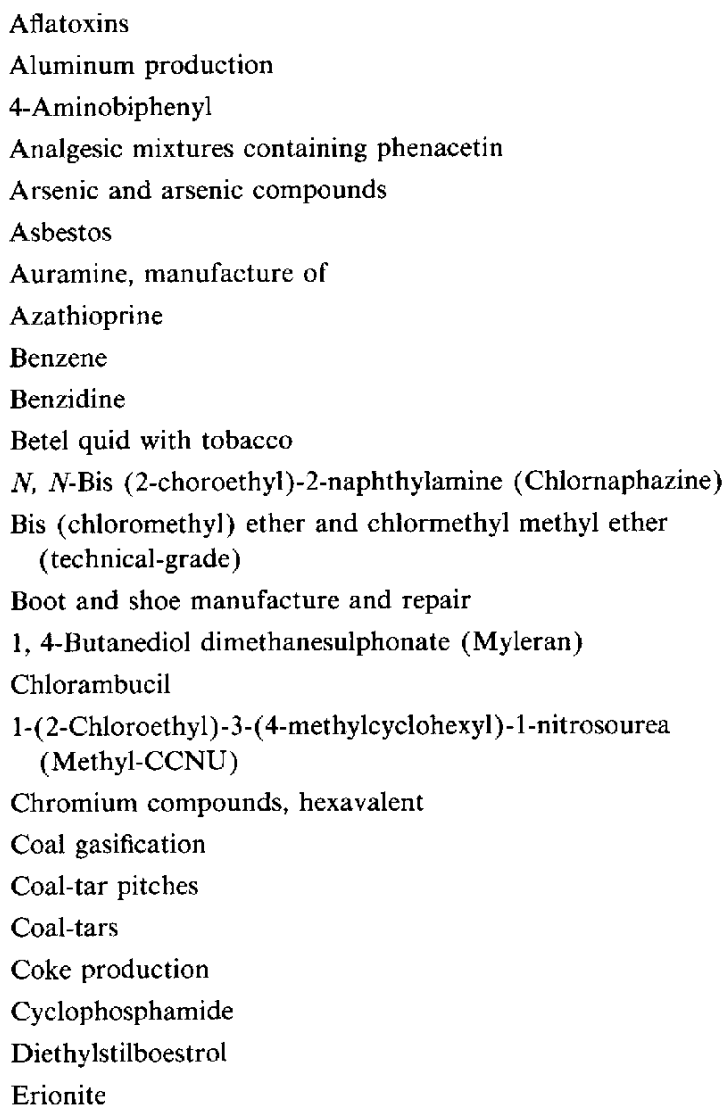

Furniture and cabinet making

Haematite mining, underground, with exposure to radon

Iron and steel founding

Isopropyl alcohol manufacture, strong-acid process

Magenta, manufacture of

Melphalan

8-Methoxypsoralen (Methoxsalen) plus ultraviolet radiation

Mineral oils, untreated and mildly-treated

MOPP (combined therapy with nitrogen mustard, vincristine, procarbazine and prednisone) and other combined chemotherapy including alkylating agents

Mustard gas (Sulphur mustard)

2-Naphthylamine

Nickel and nickel compounds

Oestrogen replacement therapy

Oestrogens, nonsteroidal

Oestrongens, steroidal

Oral contraceptives, combined

Oral contraceptives, sequential

The rubber industry

Shale-oils

Soots

Talc containing asbestiform fibres

Tobacco products, smokeless

Tobacco smoke

Treosulphan

Vinyl chloride their contribution to human cancer development may be less than a few percent ${ }^{24)}$. It could be claimed that their effect may not be additive but synergistic, but no evidence to support such an idea is yet available.

\section{Conclusion}

Based on the risk assessment of carcinogens by quantitative methods recently introduced, most environmental carcinogens are not related to human cancer development except in a few cases. The proportion of human cancers attributable to the known environmental carcinogens may be very small, even if all these agents are taken together.

On the other hand, however, human cancer development has been claimed to be closely related to environmental factors which we encounter in our daily life $\mathrm{e}^{4}$. In this context environmental factors, which are not carcinogenic themselves but which enhance or promote cancer development when given simultaneously or in different timing with carcinogenic agents, should be regarded as very important $^{24)}$. They include factors such as strong alcoholic beverages, and hot or hard food for esophageal cancer ${ }^{25}$, high-salt or hot food for stomach cancer $^{26)}$, a high-fat diet for colon and breast cancers ${ }^{27)}$, and cigarette smoking for lung or other cancers. An apparent dose-dependent increase in colon or breast cancer versus fat intake or the same trend in stomach and lung cancers related to salt and cigarette smoking, respectively, suggests that the difference in the intake of these factors is actually involved in the difference in cancer incidence in various countries or in various human populations. Many environmental carcinogens as well as oxygen radicals or ionizing radiation, which have genotoxic activity, may be working as background factors in human cancer development by affecting structural changes in cancer-related genes. But the process of complete carcinogenesis of the cells with gene alterations or clonal growth of established cancer cells may be much influenced by modifying factors in the environment. This means that we should not be overly concerned about each environmental chemical which has been proven to cause cancer in experimental animals. As for the practical purpose of primary cancer prevention, an improvement in life style so as to reduce the intake of cancer-enhancing 
factors which are commonly present as major constituents in food or other parts of the environment should be particularly important. This is especially so up to the age of 50 or 60 years, after which cancer development may be unavoidable although its timing and type may change to some extent.

\section{References}

1) Imaida K, Hasegawa $R$, Seki $K$, et al. Studies on the biological characteristics of malignant tumors in old patients. Proceedings of the 54th Annual Meeting of the Japanese Cancer Association. Kyoto, October 3 to 5, 1995, p. 538 (in Japanese).

2) Maekawa A, Onodera $\mathrm{H}$, Tanigawa $\mathrm{H}$, et al. Neoplastic and non-neoplastic lesions in aging Slc : Wistar rats. J Toxicol Sci 1983; 8: 279-290.

3) Maekawa A, Onodera H, Tanigawa H, et al. Spontaneous neoplastic and non-neoplastic lesions in aging Donryu rats. Jpn J Cancer Res 1986; $77: 882$ -890 .

4) Doll R, Peto R. The causes of cancer: Quantitative estimates of avoidable risks of cancer in the United Sates today. J Natl Cancer Inst 1981; 66: 11911308.

5) Office of Science and Technology Policy. Chemical carcinogens; A review of the science and its associated principles. Washinton DC: Office of Science and Techonology Policy, 1985.

6) Sato S. Regulation of food-related carcinogens in Japan. Reg Toxicol Pharm 1990; 11:149-157.

7) Ikeda Y. Chronic toxicity of furylfuramide to mice. Report to Food Sanitary Committee of the Ministry of Health and Welfare, Japan 1974 (in Japanese).

8) Ministry of Labor. Standards for mutation tests using microorganisms, rev. ed. Tokyo: Cental Association for Disaster Prevention, 1986.

9) Matsushima T. Chemical safety evaluation in Japan. Proceedings of Satellite Symposium of IUTOX on Toxicology Testing of Industrial Chemicals, July 26, Tokyo. 1986: 175-178.

10) National Research Council. Commission on Life Sciences. Committee on The Institutional Means for Assessment of Risks to Public Health. Risk assessment in the Federal Government: Managing the process. Washington, DC: National Academy of Sciences, 1983.

11) Ito Y. Report on the daily intake of food additives by Japanese. Tokyo: Shakai Hoken Publ Comp, 1988 (in Japanese).

12) Ito N, Fukushima S, Hagiwara A, Shibata M, Ogiso T. Carcinogenicity of butylated hydroxyanisole in $\mathbf{F}$ 344 rats. J Natl Cancer Inst 1983; 70: 343-352.

13) Hirose $M$, Shibata $M$, Hagiwara $A$, Imaida $K$, Ito $\mathrm{N}$. Chronic toxicity of butylated hyohoxytoluene in Wistar rats. Fd Cosmet Toxicol 1981; 19: 147-151.
14) Taylor JM, Weinberger MA, Friedman L. Chronic toxicity and carcinogenicity to the urinary bladder of sodium saccharin in the in utero-exposed rat. Toxicol Appl Pharmacol 1980; 54: 57-75.

15) Peto R, Pike MC, Bernstein L, Gold LS, Ames BN. The $\mathrm{TD}_{50}$ : A proposed general convention for the numerical description of the carcinogenic potency of chemicals in chronic-exposure animal experiments. Environ Health Persp 1984; 58: 1-8.

16) Food Safety Council. Quantitative risk assessment. In: Proposed system for food safety assessment, Final report of the Scientific Committee of the Food Safety Council. Washington DC: Food Safety Council, 1980: 137-160.

17) Hayashi Y, Kurokawa Y, Maekawa A. Risk evaluation of tumor-inducing substances in foods. In: Hayashi Y, Nagao M, Sugimura T, et al., eds. Diet, Nutrition and Cancer. Tokyo: Japan Sci Soc Press, 1986: 295-303.

18) Hayashi Y. Pathology of carcinogenesis by environmental chemicals; Pathological basis of primary prevention. Tr Soc Pathol Jap 1988; 77: 3-24 (in Japanese).

19) Sugimura T, Sato S, Wakabayashi K. Mutagens/carcinogens in pyrolysates of amino acids and proteins and in cooked foods: Heterocyclic aromatic amines. In: Woo Y-T, Lai DY, Arcos JC, Argus MF, eds. Chemical induction of cancer Vol. IIIC. San Diego: Academic Press, 1988: 681-710.

20) Sato S. Risk evaluation of carcinogesis. Report on the project study supported by the Ten-Year Strategy for Cancer Control, 1985. Ministry of Health and Welfare, Japan, 1986: 51-55 (in Japanese).

21) Ministry of Health and Welfare. The Japanese standard of food additives, 5th ed. Tokyo: Ministry of Health and Welfare, Japan, 1988.

22) International Agency for Research on Cancer (IARC). IARC monographs on the evaluation of carcinogenic risks to humans. Overall evaluations of carcinogenicity: An updating of IARC monographs Vols. 1 to 42. Supplement 7. Lyon: IARC, 1987.

23) Ogawa M, Mori $O$, Kamijo $T$, et al. The occurrence of acute lymphoblastic leukemia shortly after the cessation of human growth hormone therapy. Jpn J Clin Oncl 1988; 18:255-260.

24) Sato S. Cancer and dietary habits. Asian Med J $1988 ; 31: 541-547$

25) Tuyns AJ. Epidemiology of alcohol and cancer. Cancer Res 1979; 39: 2840-2843.

26) Sato T, Fukuyama $T$, Suzuki $T$, Takayanagi J, Murakami T, Tsuji R. Studies on the causation of gastric cancer. Bull Inst Public Health 1959; 8: 187-198.

27) Carroll KK, Khor HT. Dietary fat in relation to tumorigenesis. Progr Biochem Pharmacol 1975; 10: 308-353. 